\title{
Orthopedic management of mucopolysaccharide disease
}

\author{
Klane K. White ${ }^{\mathrm{a}, \mathrm{b}, *}$ and Paul Harmatz ${ }^{\mathrm{c}}$ \\ ${ }^{a}$ Seattle Children's Hospital, Department of Orthopedic Surgery, Seattle, WA, USA \\ ${ }^{\mathrm{b}}$ University of Washington, Department of Orthopedics and Sports Medicine, Seattle, WA, USA \\ ${ }^{\mathrm{c}}$ Children's Hospital \& Research Center Oakland, Oakland, CA, USA
}

\begin{abstract}
With advances in the treatment of the mucopolysaccharidosis (MPS) disorders, musculoskeletal problems are increasingly becoming a focus of care for these patients. This review discusses the current understanding of the pathophysiology of musculoskeletal disease in MPS and its orthopedic management. Deformities of the spine, hips and extremities are common and often functionally limiting. Carpal tunnel syndrome and flexor tendon triggering are common. Surgical intervention is often required to optimize long-term function.
\end{abstract}

Keywords: Mucopolysaccharidosis, orthopedics, spine, hip, knee

\section{Introduction}

The mucopolysaccharidoses (MPS) constitute a family a lysosomal storage diseases resulting from an inborn error of metabolism and subsequent abnormal accumulation of cellular glycosaminoglycans (Table 1). Mucopolysaccharides, or glycosaminoglycans (GAGs), are important constituents of the connective tissue matrix, and play a critical role in collagen-matrix adhesion, and consequently cell and tissue integrity. Abnormal accumulation of these molecules disrupts most normal organ system processes [9,22,25,29,32]. Depending on the degree of enzymatic defect, the MPS disorders can be characterized by a large spectrum of clinical involvement. Multiple organ system involvement is typical and includes central nervous system, cardiopulmonary solid organ, ocular and musculoskeletal disease. The skeletal disease manifestations range from mild platyspondyly with or without epiphyseal dysplasia to severe, life-threatening spinal deformities

\footnotetext{
* Address for correspondence: K.K. White, 4800 Sand Point Way, W-7706, Seattle, WA 98105, USA. Tel.: +1 206987 5678; Fax: +1 206987 3852; E-mail: klane.white@ seattlechildrens.org.
}

and crippling hip deformities $[9,19,47]$. Both joint stiffness and ligamentous laxity are associated with MPS disorders, compounding the problems associated with the skeletal deformities.

Dysostosis multiplex, the constellation of radiographic abnormalities classically seen in MPS, results from defective endochondral and membranous growth throughout the body, including the hips, knees and spine [5,8,31]. Additional findings include enlargement of the skull, a thick calvarium, J-shaped sella turcica, broad clavicles and ribs. The molecular physiology of this problem is not yet completely understood. Dysregulation of many structurally related cytokines has been described in MPS animal models, including MMP-13, FGF-2 and elastin binding protein [10,27, 35]. Examination of the physes from MPS I animal models and human biopsy specimens reveals growth plates that are wider than normal, with the widening being dispersed over all anatomic zones $[5,31,34]$. The quality of cells appears grossly normal in the reserve and proliferative zones, but electron microscopy reveals enlarged, vacuolated cytoplasm. The zone of hypertrophy is hypercellular and demonstrates increasing disorganization. The zone of provisional calcification 


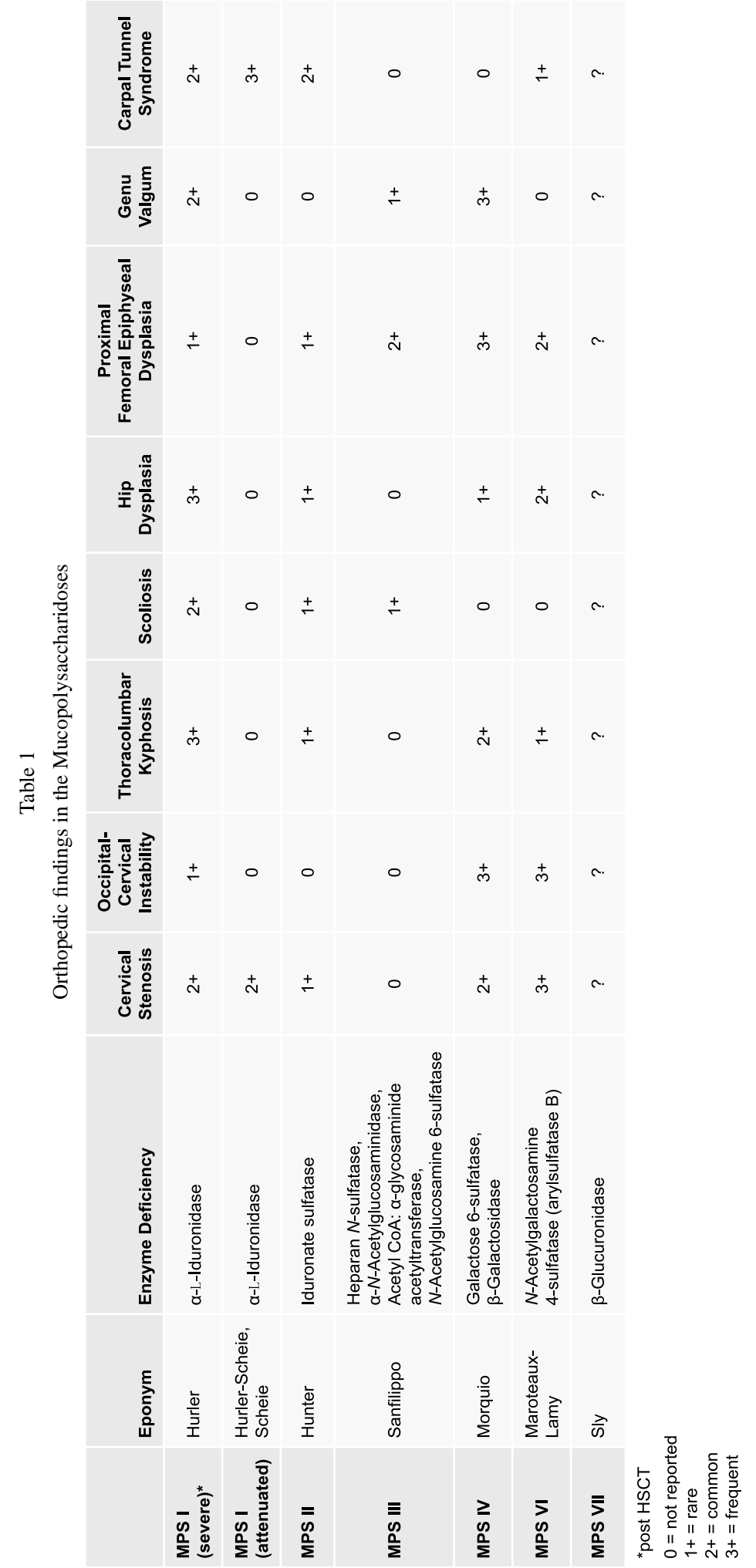




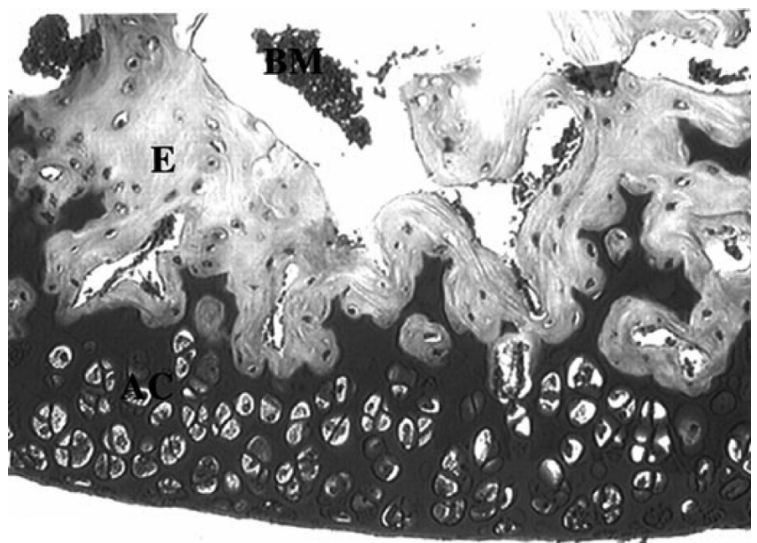

A

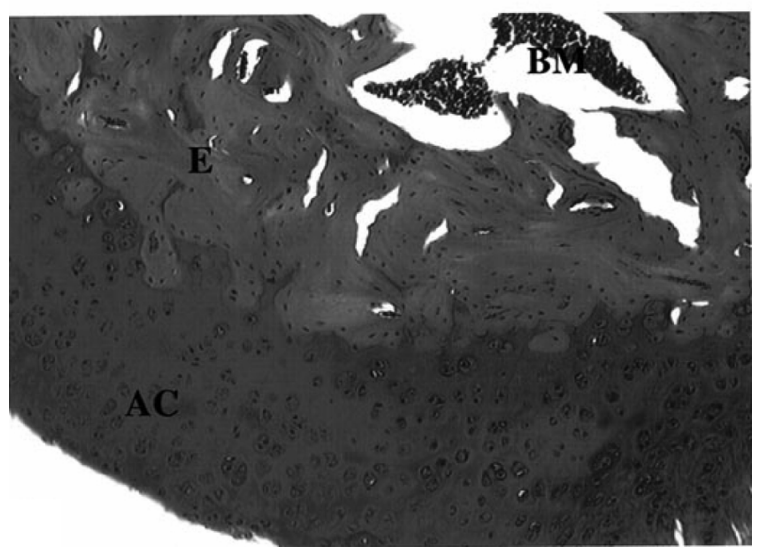

B

Fig. 1. Proteoglycan depletion in the articular cartilage of MPS VI rats. Longitudinal sections of 6-month-old MPS VI (A) and normal (B) rat proximal tibia were stained with safranin-O to visualize proteoglycans. BM, bone marrow; E, epiphyses; AC, articular cartilage. Note that the epiphyses of the MPS VI rats stained less intensely than those of normal rats of the same age. Magnification, x 40. (Figure taken from Simonaro et al., Lab Invest 81(9) (2001), 1319-1328).

is also enlarged and disorganized. Incomplete breakdown of the physeal cartilage matrix, leads to disorganized ossification with islands of remnant cartilage and erratic cortical bone formation in the primary spongiosa. These processes become more pronounced with maturity and ultimately result in the observed skeletal dysplasia [31].

Articular cartilage is also abnormal in MPS disorders. This has been demonstrated by arthroscopy in patients with MPS IVand in animal models with MPS VI [15,37]. Histopathology and biochemical analysis demonstrate proteoglycan and collagen depletion in articular cartilage matrix. This is thought to be the result of dermatan sulfate induced nitric oxide (NO) and tumor necrosis alpha (TNF- $\alpha$ ) release, and subsequent chondrocyte apoptosis [37]. These findings likely result in the subchondral delamination that has been observed arthroscopically (Fig. 1).

Because musculoskeletal deformities account for greater than $40 \%$ of the presenting signs for some MPS disorders [45], familiarity with these disorders is critical, not only for recognition and diagnosis, but for treatment of these devastating diseases. Early diagnosis allows time-sensitive medical treatment, particularly in those disorders responsive to hematopoietic stem cell transplantation (MPS IH and MPS VI) [4,17,28, 33]. Hematopoietic stem cell transplant (HSCT), while often life-saving through the alleviation of disease for the majority of major organ systems, does not alter the course of progressive skeletal disease, although some normalization of articular cartilage and linear growth may occur [5]. Enzyme replacement therapy (ERT) on the other hand, has been shown to reduce the burden of skeletal disease in MPS VI cats treated from birth [2].

Regardless of the method of specific treatment, it has become increasingly clear that children with even the most severe forms of MPS are now living into adolescence, and conceivably beyond. If left untreated, these skeletal problems usually become functionally limiting and often painful [8]. The goals of surgical procedures for the spine, hips, knees and hands should be to promote optimal function and gait. Surgical and nonsurgical management of the musculoskeletal manifestations of MPS are discussed here.

\section{Cervical spine}

Issues involving the cervical spine are extremely common in children with MPS, and may be potentially life-threatening. Atlantoaxial instability with resultant myelopathy and spastic quadriparesis is well described in MPS IV and MPS VI [12,19,40,43] (Fig. 2a, b). Quadriparesis is a known cause of premature death in affected individuals [19]. In addition, GAG accumulation behind the odontoid process may result in progressive stenosis and resultant compression of the spinal cord at the occipital-cervical junction [38]. Consequently, prophylactic fusion, often with decompression, has been recommended in all individuals affected by MPS IV [19,30]. In MPS I, growth of the odontoid process may normalize after HSCT, but enlargement of the odontoid process continues [11,13,47]. Thus, spinal instability and spinal cord compression may still occur, but may be less common. 


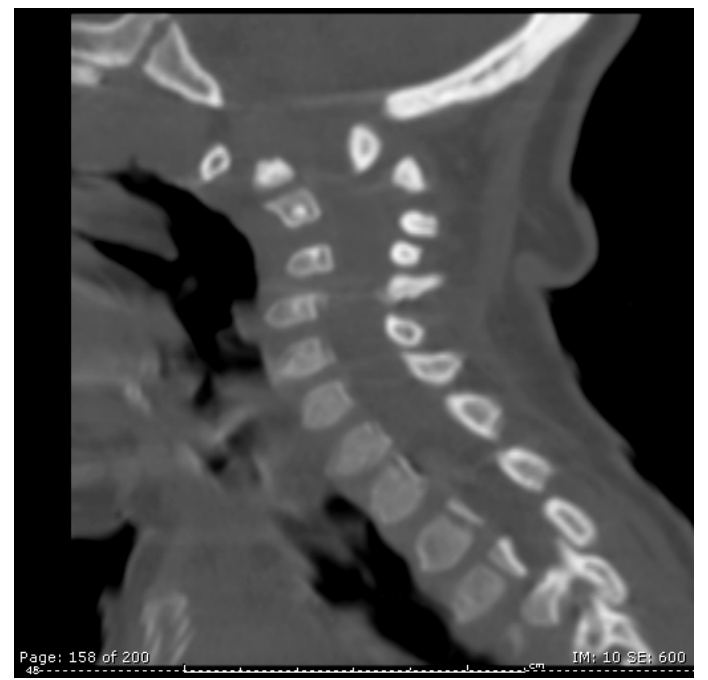

(a)

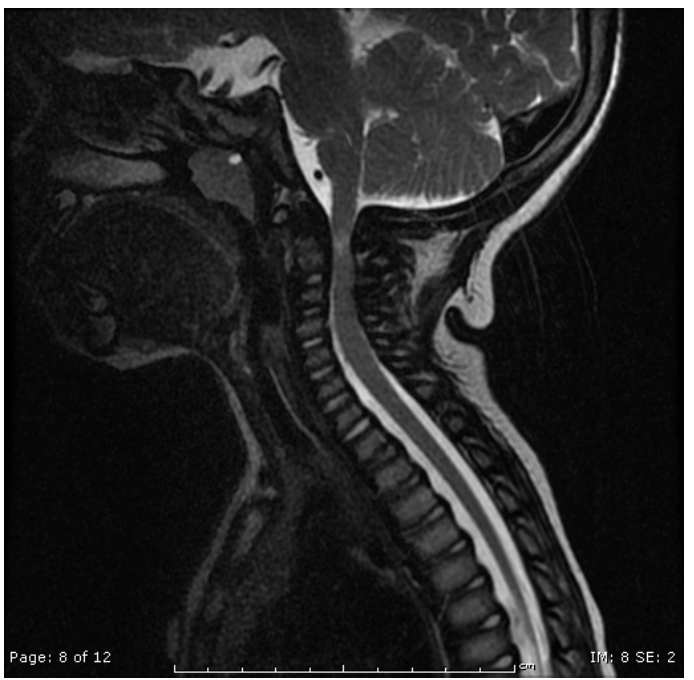

(b)

Fig. 2. CT scan (a) and MRI (b) from a two year-old child with MPS VI seen 6 months after a fall backwards from standing. The patient was partially quadriparetic, but had recovered most function at the time of evaluation. He subsequently underwent $\mathrm{C} 1 \mathrm{decompression}$ and occipital-cervical fusion. Images show anterior displacement of $\mathrm{C} 1$ on $\mathrm{C} 2$ with resultant stenosis on $\mathrm{CT}$ scan. Myelomalacia is appreciated at the same level with the posterior arch of $\mathrm{C} 1$ impinging on the spinal cord.

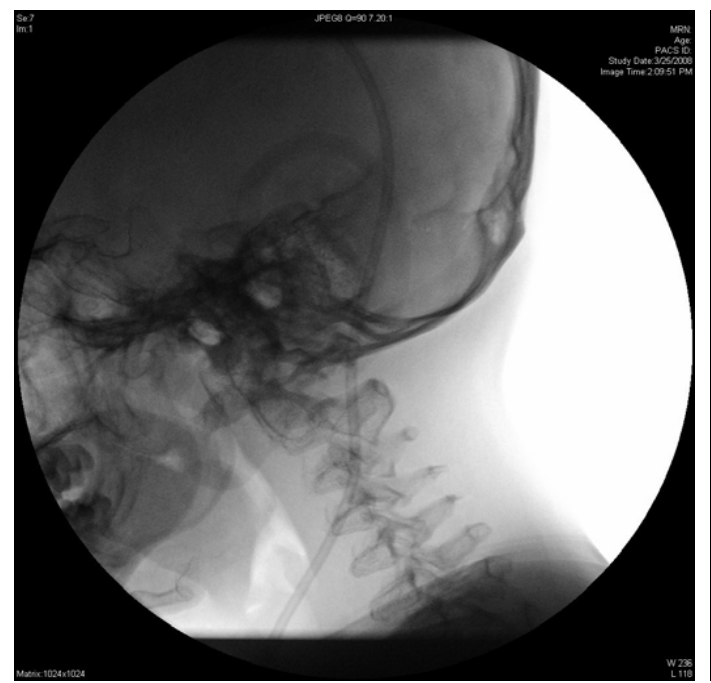

(a)

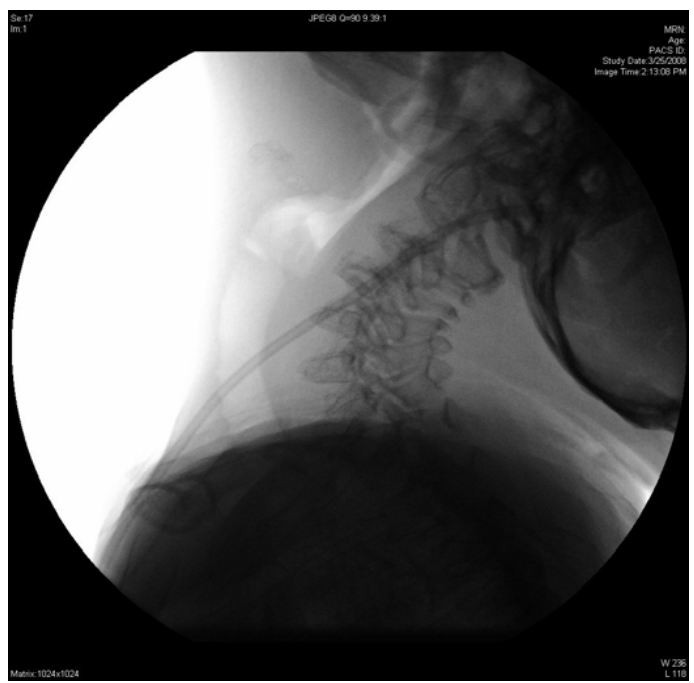

(b)

Fig. 3. Dynamic fluoroscopic images taken of the cervical spine in this eight year old MPS I patient, while awake, demonstrate subtle motion between the occiput and $\mathrm{C} 1$, and posterior widening between $\mathrm{C} 1$ and $\mathrm{C} 2$. This patient had received a matched cord blood transplant.

Atlanto-axial instability is often not demonstrated on flexion-extension cervical spine films [48]. It is unclear whether this lack of hypermobility is a failure of adequate imaging due to patient splinting. Dynamic fluoroscopy on an awake patient can unmask instability not seen on static flexion-extension plain films [49] (Fig. 3). The pathophysiology of cervical stenosis in MPS may result from delayed ossification of the odontoid pro- cess. The persistent cartilaginous anlage is prone to repetitive trauma, and at times, fracture $[30,38]$. This odontoid trauma results in two distinct problems that contribute to spinal cord injury. The first is atlanto-axial instability, which results from dens fracture and associated ligamentous instability. This is commonly demonstrated on flexion-extension radiographs. The second is cartilage/fibrocartilage reactive hypertrophy around 


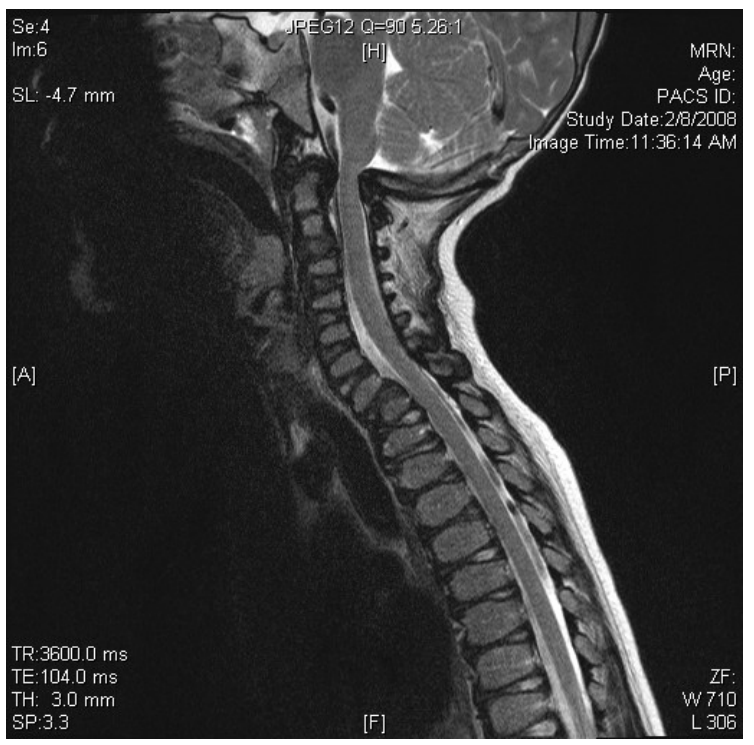

Fig. 4. An MRI from the same MPS I patient (from Fig. 4) shows significant stenosis between a retroverted odontoid process with overlying hypertrophic cartilage and the foramen magnum.

the odontoid process, as demonstrated on pathology samples by Ashraf et al. [1,24], compounded by thickening of the dura and ligamentum flavum hypertrophy, both of which have been demonstrated by imaging and pathology specimens $[13,24]$. This process results in cervical stenosis and cord compression from a combination of odontoid fibrocartilage hypertrophy, ligamentous laxity and soft tissue thickening [1,38].

Atlantoaxial instability has been reported in MPS VI (Maroteaux-Lamy) and in severe, untreated MPS I (Hurler) $[6,42,43]$. Children with MPS I, who have undergone hematopoietic stem cell transplant (HSCT) often have normalization of their odontoid process [11]. Many of these patients however continue to demonstrate deposition of GAGs in the subarachnoid space directly behind the $\mathrm{C} 2$ vertebra $[11,13]$, and some have required surgical treatment of cervical spine disease after HSCT (Fig. 4). Even the more attenuated forms of MPS I (Hurler-Scheie and Scheie) develop surgicalgrade cervical stenosis with myelopathy in the upper cervical spine and require careful monitoring for this process [45].

Multilevel cervical stenosis has also been reported in MPS VI (Maroteaux-Lamy), in addition to atlantoaxial instability [1]. Imaging in these patients reveals posteriorly prominent intervertebral discs, thickened dura and hypertrophy of the ligamentum flavum. This finding is also demonstrated in the MPS VI cat model (Mark Haskins, personal communication). Pathology

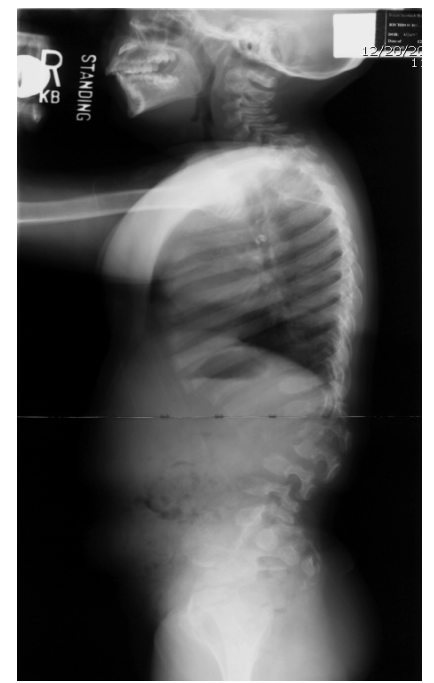

(a)

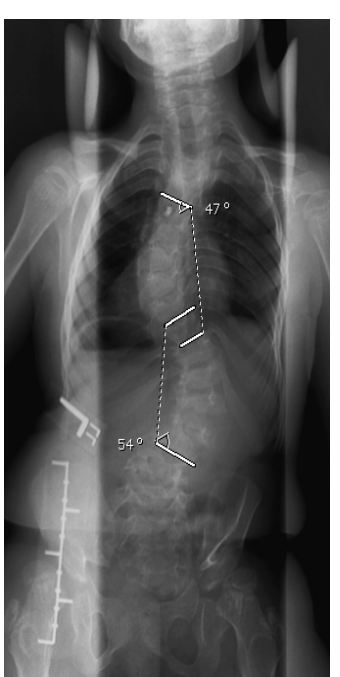

(b)
Fig. 5. A typical thoracolumbar kyphosis (a) is seen in this nine year old girl with MPS I after matched unrelated bone marrow transplantation. She also developed a significant double major scoliosis. She continues to be observed without any interventions.

samples taken at the time of durotomy show the classic MPS cellular pathology with cells filled with enlarged lysosomes due to GAG accumulation [13]. In the absence of instability, cervical decompression without fusion may be appropriate.

Several authors have recommended prophylactic decompression and fusion of the occipital cervical junction at an early age for MPS IV [19,30,38]. Current recommendations are for decompression and fusion of asymptomatic patients when the space available for the cord is $<14 \mathrm{~mm}$ or there is cervical instability $>8 \mathrm{~mm}$. Patients with 5 to $8 \mathrm{~mm}$ of cervical instability, with clinical evidence of spinal cord impingement, and all patients with a deteriorating neurological condition also warrant surgical treatment [48].

It is recommended that all children with MPS should avoid "high risk" activities such as contact sports, gymnastics, etc. In addition, these children should be treated with caution when undergoing positioning for anesthesia, and at least one set of flexion and extension lateral radiographs of the cervical spine is recommended prior to anesthesia in all affected individuals to evaluate for atlantoaxial instability.

\section{Thoracolumbar spine}

The hallmark orthopedic feature in MPS is thoracolumbar kyphosis or the gibbus deformity, and has 
historically played a significant role in the diagnosis of MPS [3,45]. Gibbus deformities occur in nearly all children with severe MPS I, and are commonly found in other MPS disorders [8] (Fig. 5a). The kyphosis develops from poor bone growth in the anterior-superior aspect of the upper lumbar vertebrae. This process results in anterior wedging, retrolisthesis of the apex vertebrae and anterior herniation of the intervetebral discs at the thoracolumbar junction [18]. Surgical stabilization to halt the progression of the kyphosis is relatively common in MPS I and in MPS VI, even after HSCT [8, 9,47]. Scoliosis has been observed in MPS I, II, III, but is rarely significant enough to require surgery [9] (Fig. 5b).

There is no clinical evidence to support the use of bracing in MPS. Bracing may slow the progression of both spinal kyphosis and scoliosis, delaying surgery, but not preventing surgery. Bracing can be uncomfortable for children, and they rarely tolerate it, especially young children due to their stature and abdominal girth. Consequently, bracing is only recommended in young children with progressive deformity who are not otherwise candidates for surgery. One must consider the psychosocial implications for the patients and their families.

Indications for surgery vary, depending on the needs of the child and the desires of the family. Generally, a kyphosis of more than $70^{\circ}$ or scoliosis greater than $50^{\circ}$ are relative indications for surgery. (James Ogilvie, personal communication) The presence of myelopathy is clearly an indication for surgery. Often the medical complexity of these patients can be a factor against surgical intervention. Surgery for kyphosis or scoliosis may be necessary as young as two years of age, and usually before adolescence. Published reports put the average age at about eight years [8]. Current experience suggests that, if possible, delaying spinal surgery allows maximal growth of the spine and further development of already osteopenic and small, dysplastic bone, thus reducing the technical difficulty of the procedure.

Anterior and posterior fusion for kyphotic deformities is recommended, followed by postoperative bracing for 3-6 months. Early experience suggests that treatment of kyphosis by posterior instrumentation and fusion only may lead to an increased risk of failure and the need for re-operation. (James Ogilvie, personal communication) In general, when these guidelines are followed, there does not seem to be in increased risk of pseudoarthrosis (failure for bones to fuse) in this patient population. Treatment of scoliosis follows traditional indications for intervention in complex defor-

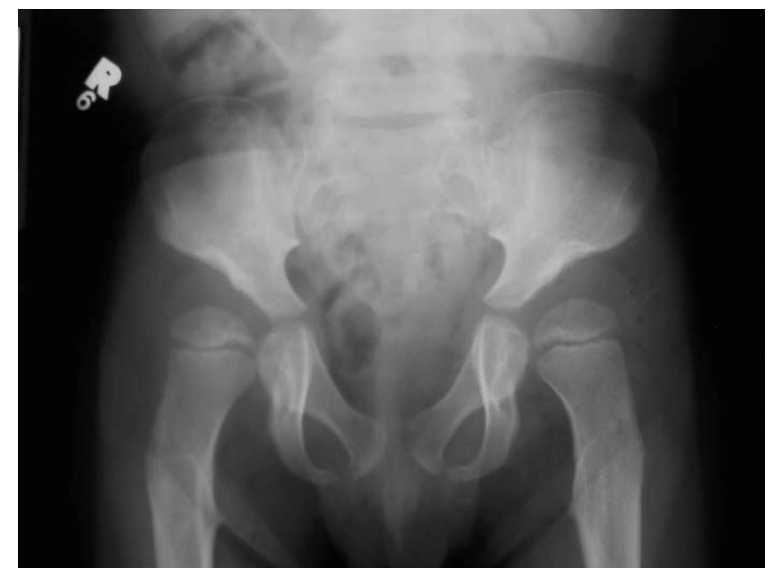

(a)

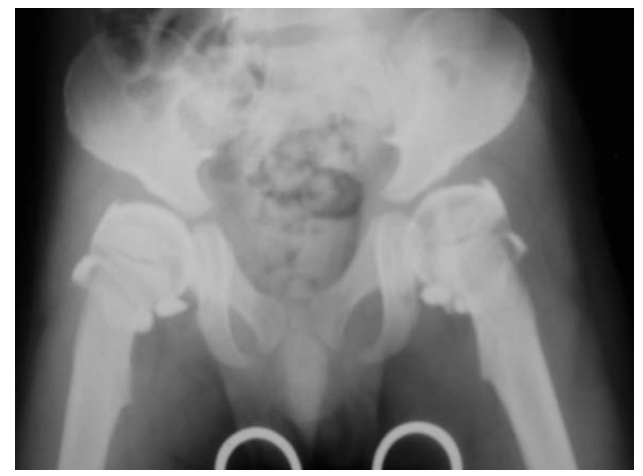

(b)

Fig. 6. An anteroposterior view of the hips (a) in a patient with MPS I, after matched unrelated bone marrow transplantation, demonstrates significant hip dysplasia with a poorly developed acetabulum, coxa valga, and apparent subluxation of the femoral heads. An arthrogram taken three years previously demonstrates a well developed cartilaginous enlage which failed to ossify.

mity, including the recommendation for anterior fusion in less mature children in order to prevent crankshaft phenomenon.

\section{Hips/pelvis}

Hip concerns are present in nearly all individuals with MPS, and can be divided into two major categories: hip dysplasia and osteonecrosis-like epiphyseal dysplasia $[8,16,20,41,47]$. In MPS-related hip dysplasia there is a poorly developed acetabulum, underdevelopment of the medial portion of the proximal femoral epiphysis, and coxa valga. There appears to be delayed ossification of the lateral acetabular corner, leaving a significant cartilaginous anlage as demonstrated by arthrogram (Fig. 6a, b). In our experience, MRI images confirm these arthrography findings. This com- 
bination of bone defects results in progressive hip instability and late dislocation. This can be functionally limiting by adolescence or early adulthood [8].

Epiphyseal dysplasia, which has the appearance of osteonecrosis, or Perthes Disease, is seen in MPS III, IV and VI (Fig. 7a). This process may represent an inflammatory mediated apoptosis [36]. Surgical reconstruction will not correct these deformities; however the role of containment surgery has not been investigated, and may be appropriate in selected cases. Ultimately, prosthetic replacement of the hip may be required (Fig. 7b). This is an extremely challenging procedure in patients with MPS, and should be reserved for individuals with incapacitating hip pain, and performed by surgeons versed in complex hip reconstructive surgery. In the future, early medical treatment with intra-articular anti-inflammatory medications may reduce the theoretical apoptosis and subsequent osteonecrosis-like processes [35].

Hip dysplasia to some degree is found in nearly all children with severe MPS I, and can also be found in children with attenuated MPS I, MPS II, MPS VI, and less often MPS IV $[16,41,49]$. Abduction bracing in young children with MPS has not been studied, but is likely ineffective and may actually result in worsening muscle weakness and delay of physical development. Hip dysplasia has not been shown to respond to stem cell transplant or enzyme replacement therapy although very early treatment after birth has not been well studied, and most children with MPS I after HSCT eventually require corrective hip surgery. Surgery on the hips is performed more easily at a younger age, around age 5-7, for an optimal outcome, but has been reported in children as young as 2 years of age. Technically, successful surgery becomes much more difficult at older ages (after triradiate cartilage closure).

Surgical reconstruction for hip dysplasia (primarily in MPS I) is a combination of osteotomies intended to reposition the bones and optimize hip mechanics [16, 41]. A pelvic osteotomy such as described by Salter, or Dega (as modified by Mubarak et al.) is required to reduce the global acetabular deficiency $[21,46]$. A proximal femoral osteotomy is also required to reduce the significant valgus deformity of the femoral neck. On occasion, capsulorrhaphy is required when the hip is dislocated. It should be noted that the hip capsule can be extremely thick and difficult to work with (up to $10 \mathrm{~mm}$ thick, personal communications, James Oglvie) Without hip surgery, there may be progressive pain and stiffness, and eventually frank dislocation of the hips, with a dramatic reduction in walking ability [8]. Thus far, the results of hip surgery in MPS are promising, resulting in improved motion and independent walking.

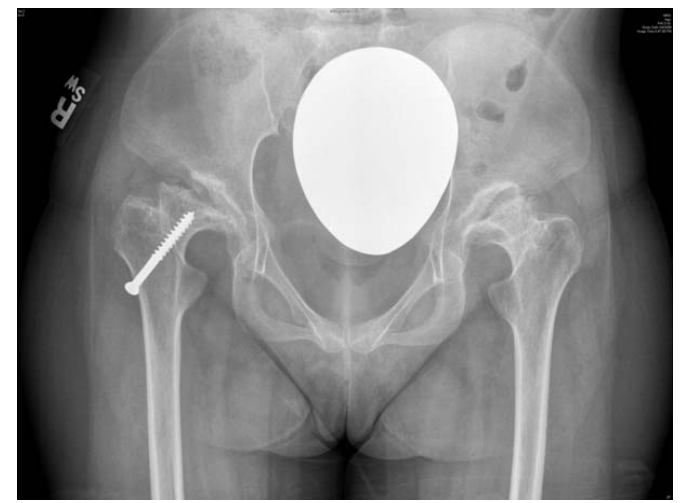

(a)

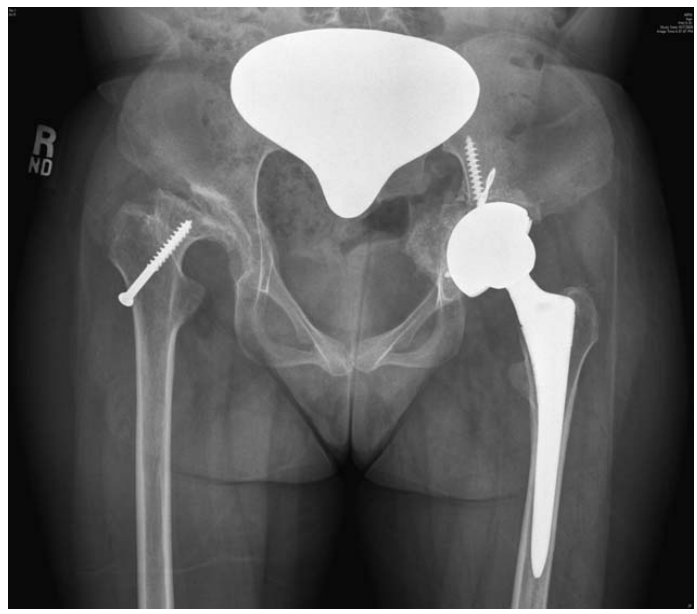

(b)

Fig. 7. Severe epiphyseal dysplasia with erosion and collapse of the femoral heads and subsequent subluxation in a twenty-four year old woman with MPS IV. She sustained a right femoral neck fracture which was stabilized with a screw (a). Due to severe pain symptoms, she underwent left total hip arthroplasty (b).

\section{Knees}

Almost all children with MPS IV, and about $50 \%$ of children with MPS I post-HSCT, develop genu valgum severe enough to require surgery $[7,22,26]$. Children with MPS VI as well as the attenuated forms of MPS I, can also develop knock-knees severe enough to warrant surgery. The published indication for surgery is a tibial-femoral angle greater than $15^{\circ}$. Hemiepiphyseal growth modulation through the use of Blount's staples may be performed through a relatively small incision [7, 26] (Fig. 8). A newer technology, the tension band plate ("8-plate", Orthofix, Verona, Italy), is showing great promise as an alternative to staples, particularly in children too small for staple placement [39]. Occasionally, these implants can dislodge. When this happens, they are typically removed, and if necessary, new ones 


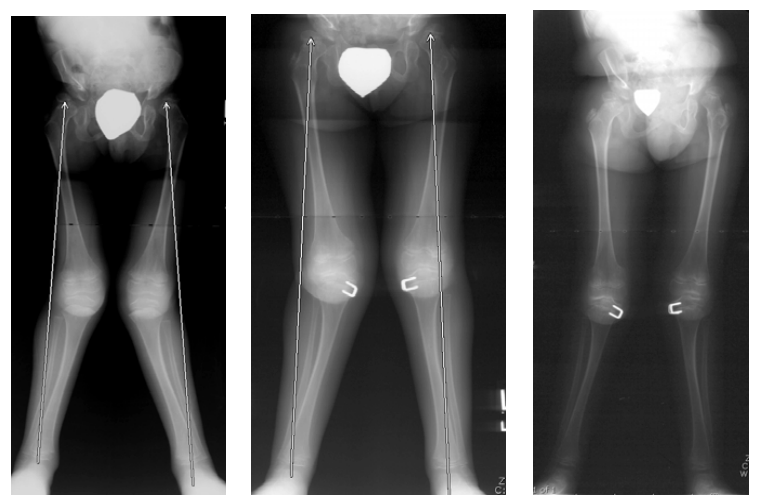

Fig. 8. The patient from Figure 6 also had significant genu valgum and had Blount's staples placed for growth modulation. In the time period covered, she showed progressive improvement of her deformity as demonstrated by the decrease in medial deviation from the mechanical axis (shown as white arrows). Surgical intervention has been recommended when the tibiofemoral angle exceeds $15^{\circ}$ (also shown in first figure to the left $-\alpha$ ).

replace them. Osteotomies near the knee (tibia or femur) may be required. Although osteotomies are more invasive and painful, staples or tension band plates will not work in children who are physically too small to have them placed, or those who do not have enough growth remaining to modulate. Growth in MPS can be difficult to predict, and as such criteria for intervention can not necessarily follow those of normal children. Experience with hip surgery has shown that children with MPS heal osteotomies well. Advanced arthrosis of the knee due to delamination of articular cartilage, in adults with MPS, may be addressed with total knee arthroplasty [7].

Children with MPS also suffer from stiff knees, which prevent full straightening and result in a crouched gait. Knee stiffness is improved with stem cell transplant and enzyme replacement therapy, but most children still require continued physical therapy to optimize knee motion and walking function [14,23]. For those with fixed knee flexion contractures, extension osteotomy of the distal femur should be considered.

\section{Upper extremities}

Upper extremity function may be adversely altered due to restricted joint motion, bony abnormalities, compression neuropathies or tenosynovial thickening. ERT has proven to be extremely beneficial in improving joint range of motion of the shoulders and elbows [14, 23]. The full benefits of ERT in this regard may take

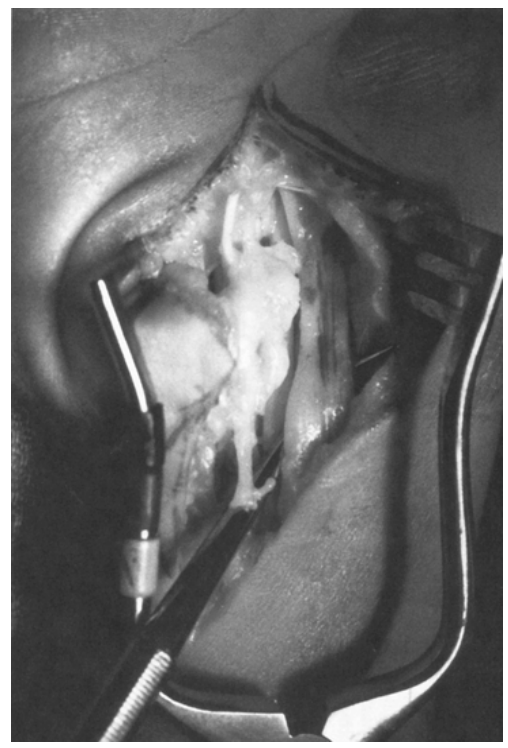

Fig. 9. Intraoperative view of a carpal tunnel decompression in a child with MPS I. Note the hourglass appearance of the median nerve adjacent to the large soft tissue accumulation sitting in the left half of the incision.

several years, however, to manifest themselves. Forearm pronation and supination are often limited by a Madelung's type deformity (most often seen in MPS II) [49]. Carpal tunnel syndrome, either due to primary neuropathy or secondarily from tenosynovial accumulation of GAGs, is well described in MPS disorders [44,50]. Median nerve neuropathy frequently goes unrecognized in children with limited expressive skills resulting in permanent loss of nerve function. As such, regular electrodiagnostic studies (nerve conduction velocities performed every one to two years) are recommended in children with MPS. Carpal tunnel release is required when median nerve disease exists or is suspected. Tenosynovial accumulation of GAGs also is known to result in "trigger digits" [44]. Surgical release is recommended when this condition exists as well, and can be done concomitantly with carpal tunnel release (Fig. 9). Delayed treatment of trigger digits may result in fixed flexion contractures.

\section{Summary}

Even with stem cell transplant or enzyme replacement therapy, patients with MPS continue to have significant muscular and skeletal disabilities, most commonly involving the spine, hips, knees and hands. These are rarely catastrophic or life threatening, but 
frequently limit a child's function, activity, and quality of life. Surgical intervention is often required, to optimize long-term function. The timing and type of surgery may vary among children and among surgeons. Regardless, early evaluation is critical in determining what treatments will be necessary to optimize the quality of life for a child with MPS.

\section{Acknowledgements}

This manuscript was developed as the result of a meeting of experts entitled "Promoting Bone Health in MPS VI: Framing New Therapies" held in Oakland, California in October, 2008. This meeting was supported by an educational grant from BioMarin Pharmaceutical, Inc., Novato, CA. BioMarin had no role in the content presented and discussed at the meeting. Editorial assistance kindly was provided by Drs. Helen Nicely and Sean Turbeville, BioMarin employees. BioMarin reviewed the manuscript to insure the accuracy of all statements regarding enzyme replacement therapy with galsulfase. All authors participated in the development and writing of the manuscript and are fully responsible for its content.

\section{Conflicts of interest}

Klane White has received honoraria for educational lectures from BioMarin Pharmaceutical Inc., and Shire plc. Paul Harmatz has provided consulting support and received speaker's honoraria and travel support from BioMarin.

\section{References}

[1] J. Ashraf et al., Transoral decompression and posterior stabilisation in Morquio's disease, Arch Dis Child 66(11) (1991), 1318-1321.

[2] D. Auclair et al., Replacement therapy in Mucopolysaccharidosis type VI: advantages of early onset of therapy, Mol Genet Metab 78(3) (2003), 163-174.

[3] P.J. Belmont, Jr. and D.W. Polly, Jr., Early diagnosis of Hurler's syndrome with the aid of the identification of the characteristic gibbus deformity, Mil Med 163(10) (1998), 711714.

[4] K.J. Bjoraker et al., Long-term outcomes of adaptive functions for children with mucopolysaccharidosis I (Hurler syndrome) treated with hematopoietic stem cell transplantation, $J$ Dev Behav Pediatr 27(4) (2006), 290-296.

[5] M.A. Breider, R.M. Shull and G. Constantopoulos, Longterm effects of bone marrow transplantation in dogs with mucopolysaccharidosis I, Am J Pathol 134(3) (1989), 677-692.
[6] C.B. Brill et al., Spastic quadriparesis due to C1-C2 subluxation in Hurler syndrome, J Pediatr 92(3) (1978), 441-443.

[7] M.C. de Waal Malefijt, A. van Kampen and J.J. van Gemund, Total knee arthroplasty in patients with inherited dwarfisma report of five knee replacements in two patients with Morquio's disease type A and one with spondylo-epiphyseal dysplasia, Arch Orthop Trauma Surg 120(3-4) (2000), 179182.

[8] R.E. Field et al., Bone-marrow transplantation in Hurler's syndrome. Effect on skeletal development, J Bone Joint Surg Br 76(6) (1994), 975-981.

[9] J.A. Herring, Skeletal Dysplasias Mucoplysaccharisosis, (3ed Tachjian), (Vol. 3), J. Herring, ed., Philadelphia, W.B Saunders, 2002, PP. 1775-1793.

[10] A. Hinek and S.E. Wilson, Impaired elastogenesis in Hurler disease: dermatan sulfate accumulation linked to deficiency in elastin-binding protein and elastic fiber assembly, Am J Pathol 156(3) (2000), 925-938.

[11] S.H. Hite C. Peters and W. Krivit, Correction of odontoid dysplasia following bone-marrow transplantation and engraftment (in Hurler syndrome MPS 1H), Pediatr Radiol 30(7) (2000), 464-470.

[12] D.G. Hughes et al., MRI of the brain and craniocervical junction in Morquio's disease, Neuroradiology 39(5) (1997), 381385.

[13] E. Kachur and R. Del Maestro, Mucopolysaccharidoses and spinal cord compression: case report and review of the literature with implications of bone marrow transplantation, $\mathrm{Neu}$ rosurgery 47(1) (2000), 223-228; discussion 228-229.

[14] E.D. Kakkis et al., Enzyme-replacement therapy in mucopolysaccharidosis I, N Engl J Med 344(3) (2001), 182-188.

[15] T. Kalteis et al., Arthroscopic and histologic findings in Morquio's syndrome, Arthroscopy 21(2) (2005), 233-237.

[16] T. Kanazawa et al., Femoral head dysplasia in Morquio disease type A: bilateral varus osteotomy of the femur, Acta Orthop Scand 72(1) (2001), 18-21.

[17] W. Krivit, C. Peters and E.G. Shapiro, Bone marrow transplantation as effective treatment of central nervous system disease in globoid cell leukodystrophy, metachromatic leukodystrophy, adrenoleukodystrophy, mannosidosis, fucosidosis, aspartylglucosaminuria, Hurler, Maroteaux-Lamy, and Sly syndromes, and Gaucher disease type III, Curr Opin Neurol 12(2) (1999), 167-176.

[18] T.L. Levin et al., Lumbar gibbus in storage diseases and bone dysplasias, Pediatr Radiol 27(4) (1997), 289-294.

[19] S.J. Lipson, Dysplasia of the odontoid process in Morquio's syndrome causing quadriparesis, J Bone Joint Surg Am 59(3) (1977), 340-344.

[20] E.L. Masterson et al., Hip dysplasia in Hurler's syndrome: orthopaedic management after bone marrow transplantation, J Pediatr Orthop 16(6) (1996), 731-733.

[21] N.P. McNerney, S.J. Mubarak and D.R. Wenger, One-stage correction of the dysplastic hip in cerebral palsy with the San Diego acetabuloplasty: results and complications in 104 hips, J Pediatr Orthop 20(1) (2000), 93-103.

[22] A.M. Montano et al., International morquio a registry: clinical manifestation and natural course of morquio a disease, $J$ Inherit Metab Dis 30(2) (2007), 165-174.

[23] J. Muenzer et al., A phase I/II clinical trial of enzyme replacement therapy in mucopolysaccharidosis II (Hunter syndrome), Mol Genet Metab 90(3) (2007), 329-337.

[24] M. Mut et al., Multilevel myelopathy in Maroteaux-Lamy syndrome and review of the literature, Clin Neurol Neurosurg 107(3) (2005), 230-235. 
[25] J. Nelson, Incidence of the mucopolysaccharidoses in Northern Ireland, Hum Genet 101(3) (1997), 355-358.

[26] E. Odunusi et al., Genu valgum deformity in Hurler syndrome after hematopoietic stem cell transplantation: correction by surgical intervention, J Pediatr Orthop 19(2) (1999), 270-274.

[27] C. Pan et al., Functional abnormalities of heparan sulfate in mucopolysaccharidosis-I are associated with defective biologic activity of FGF-2 on human multipotent progenitor cells, Blood 106(6) (2005), 1956-1964.

[28] C. Peters et al., Hurler syndrome: II. Outcome of HLAgenotypically identical sibling and HLA-haploidentical related donor bone marrow transplantation in fifty-four children. The Storage Disease Collaborative Study Group, Blood 91(7) (1998), 2601-2608.

[29] R. Pinto et al., Prevalence of lysosomal storage diseases in Portugal, Eur J Hum Genet 12(2) (2004), 87-92.

[30] A.O. Ransford et al., Occipito-atlanto-axial fusion in MorquioBrailsford syndrome. A ten-year experience, J Bone Joint Surg Br 78(2) (1996), 307-313.

[31] C. Russell et al., Murine MPS I: insights into the pathogenesis of Hurler syndrome, Clin Genet 53(5) (1998), 349-361.

[32] C. Scriver, A. Beaudet, S.W. and D. Valle, The Metabolic and Molecular Basesis of Inherited Disease, in: The Mucopolysaccharidoses, E.F. Neufeld, ed., Muenzer J8th 2008, New York, McGraw-Hill.

[33] E.G. Shapiro et al., Neuropsychological outcomes of several storage diseases with and without bone marrow transplantation, J Inherit Metab Dis 18(4) (1995), 413-429.

[34] C.P. Silveri et al., Hurler syndrome with special reference to histologic abnormalities of the growth plate, Clin Orthop Relat Res (269) (1991), 305-311.

[35] C.M. Simonaro et al., Joint and bone disease in mucopolysaccharidoses VI and VII: identification of new therapeutic targets and biomarkers using animal models, Pediatr Res 57(5 Pt 1) (2005), 701-707.

[36] C.M. Simonaro et al., Mechanism of glycosaminoglycanmediated bone and joint disease: implications for the mucopolysaccharidoses and other connective tissue diseases, $\mathrm{Am}$ J Pathol 172(1) (2008), 112-122.

[37] C.M. Simonaro, M.E. Haskins and E.H. Schuchman, Articular chondrocytes from animals with a dermatan sulfate storage disease undergo a high rate of apoptosis and release nitric oxide and inflammatory cytokines: a possible mechanism under- lying degenerative joint disease in the mucopolysaccharidoses, Lab Invest 81(9) (2001), 1319-1328.

[38] J.M. Stevens et al., The odontoid process in MorquioBrailsford's disease. The effects of occipitocervical fusion, $J$ Bone Joint Surg Br 73(5) (1991), 851-858.

[39] P.M. Stevens, Guided growth for angular correction: a preliminary series using a tension band plate, J Pediatr Orthop 27(3) (2007), 253-259.

[40] O. Svensson and S. Aaro, Cervical instability in skeletal dysplasia. Report of 6 surgically fused cases, Acta Orthop Scand 59(1) (1988), 66-70.

[41] C. Taylor et al., Mobility in Hurler syndrome, J Pediatr Orthop 28(2) (2008), 163-168

[42] S.L. Thomas, M.H. Childress and B. Quinton, Hypoplasia of the odontoid with atlanto-axial subluxation in Hurler's syndrome, Pediatr Radiol 15(5) (1985), 353-354.

[43] J.A. Thorne et al., Craniovertebral abnormalities in Type VI mucopolysaccharidosis (Maroteaux-Lamy syndrome), Neurosurgery 48(4) (2001), 849-852; discussion 852-853.

[44] A.E. Van Heest et al., Surgical treatment of carpal tunnel syndrome and trigger digits in children with mucopolysaccharide storage disorders, J Hand Surg [Am] 23(2) (1998), 236-243.

[45] S. Vijay and J.E. Wraith, Clinical presentation and follow-up of patients with the attenuated phenotype of mucopolysaccharidosis type I, Acta Paediatr 94(7) (2005), 872-877.

[46] J.H. Wedge, S.R. Thomas and R.B. Salter, Outcome at fortyfive years after open reduction and innominate osteotomy for late-presenting developmental dislocation of the hip. Surgical technique, J Bone Joint Surg Am 90(Suppl 2 Pt 2) (2008), 238-253.

[47] J.S. Weisstein et al., Musculoskeletal manifestations of Hurler syndrome: long-term follow-up after bone marrow transplantation, J Pediatr Orthop 24(1) (2004), 97-101.

[48] K.K. White S. Steinman and S.J. Mubarak, Cervical stenosis and spastic quadriparesis in morquio disease (MPS IV). A case report with twenty-six-year follow-up, J Bone Joint Surg Am 91(2) (2009), 438-442.

[49] K.K. White, H.S., M. Raff and M.J. Goldberg, Musculoskeletal Health in MPS II: Pediatric Outcomes Data Collection Instrument (PODCI) Demonstrates Functional Improvements in Patients on ERT, in World Symposium, 2009, San Diego, CA.

[50] A. Yuen et al., Carpal tunnel syndrome in children with mucopolysaccaridoses, J Child Neurol 22(3) (2007), 260-263. 